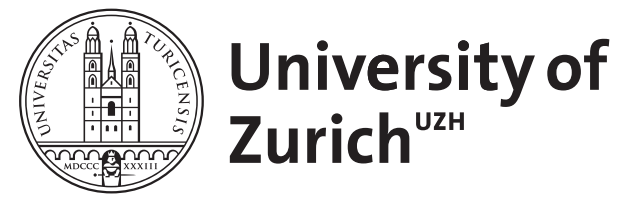

Zurich Open Repository and Archive

University of Zurich

University Library

Strickhofstrasse 39

CH-8057 Zurich

www.zora.uzh.ch

Year: 2010

Postmortale Organspende: es spricht mehr für die Zustimmungslösung als für die Widerspruchslösung

Pfister, E

Posted at the Zurich Open Repository and Archive, University of Zurich

ZORA URL: https://doi.org/10.5167/uzh-41426

Journal Article

Originally published at:

Pfister, E (2010). Postmortale Organspende: es spricht mehr für die Zustimmungslösung als für die Widerspruchslösung. Bioethica Forum, 3(2):68-69. 


\section{Postmortale Organspende: Es spricht mehr für die Zustimmungslösung als für die Widerspruchslösung}

Standpunkt

a Institut für Biomedizinische Ethik, Universität Zürich

\section{Postmortale Organspende: Zustimmungsmodelle}

Die Entnahme von Organen, Geweben und Zellen nach dem Tod kann rechtlich unterschiedlich geregelt werden. Es gibt grundsätzlich zwei verschiedene Zustimmungsmodelle, die heute in vielen Ländern angewendet werden:

1. Die Zustimmungslösung erfordert für eine Entnahme prinzipiell das aktive Einverständnis der betroffenen Person oder der Angehörigen und wird nochmals in zwei Modelle unterschieden [1]:

1.1. Die enge Zustimmungslösung erlaubt die Entnahme von Organen, Geweben und Zellen nur dann, wenn die verstorbene Person dieser zu Lebzeiten zugestimmt hat. Liegt keine Zustimmung zu einer Entnahme vor, so wird dies als Ablehnung gewertet.

1.2. Die erweiterte Zustimmungslösung sieht in einer fehlenden Erklärung der verstorbenen Person lediglich eine Nichterklärung. In diesem Fall werden deshalb die nächsten Angehörigen angefragt. Wenn die nächsten Angehörigen einer Entnahme zustimmen, so ist diese zulässig. Der Wille der verstorbenen Person hat aber - falls er vorliegt - in jedem Fall Vorrang gegenüber demjenigen der Angehörigen.

2. Die Widerspruchslösung wertet ein Schweigen als Einverständnis und erfordert für die Nichtentnahme einen expliziten Widerspruch. Sie wird nochmals in drei Modelle unterschieden [1]:

2.1. Nach der engen Widerspruchslösung dürfen Organe, Gewebe oder Zellen einer verstorbenen Person entnommen werden, wenn sich diese zu Lebzeiten nicht gegen einen solchen Eingriff ausgesprochen hat.

2.2. Die erweiterte Widerspruchslösung berücksichtigt auch die nächsten Angehörigen als Vertreter des Willens der verstorbenen Person, welche über eine Entnahme bestimmen können. In einigen Ländern gibt es eine Widerspruchsfrist für die Angehörigen.

2.3. Fehlt eine Willensäusserung der verstorbenen Person, müssen gemäss der Informationslösung die nächsten Angehörigen über eine mögliche Entnahme innerhalb einer bestimmten Frist informiert und auf ein eventuelles Widerspruchsrecht aufmerksam gemacht werden. Widersprechen diese innerhalb einer bestimmten Frist nicht, dürfen Organe, Gewebe oder Zellen entnommen werden.

\section{Die Situation in der Schweiz}

Die Entnahme von Organen, Geweben und Zellen ist in der Schweiz durch Artikel 8 des Bundesgesetzes vom 8. Oktober 2004 über die Transplantation von Organen, Geweben und Zellen rechtlich geregelt und unterliegt demgemäss der erweiterten Zustimmungslösung [2]. Seit einiger Zeit werden in der Schweiz einzelne Stimmen laut, die sich für einen Regimewechsel von der Zustimmungslösung zur Widerspruchslösung aussprechen [3]. Beispiele dafür sind die Stiftung Swisstransplant [4] und ein parlamentarisches Postulat [5], welches schliesslich vom Bundesrat abgelehnt und vom Nationalrat abgeschrieben wurde. In seiner ablehnenden Antwort hielt der Bundesrat fest, dass das Thema im Jahr 2012 drängender werden könnte, wenn die Evaluation des Bundesgesetzes über die Transplantation abgeschlossen und veröffentlicht werden wird. Es scheint deshalb jetzt sinnvoll, einen genaueren Blick auf die Frage zu werfen, ob das Zustimmungsmodell tatsächlich zugunsten eines Widerspruchmodells aufgegeben werden sollte.

\section{Widerspruchslösung: Mehr verfügbare Spenderorgane?}

Das Hauptargument der Befürworter einer Widerspruchslösung liegt in der Hoffnung auf eine grössere Anzahl verfügbarer Spenderorgane. In der Folge würden erstens weniger Menschen auf der Warteliste sterben und zweitens könnten möglicherweise Gesundheitskosten eingespart werden. Gemäss Franz Immler, Direktor von Swisstransplant, sind die Kosten für eine Nierentransplantation mit einmaligen 75000 Franken deutlich tiefer als für eine Dialyse mit ungefähr 80000 Franken pro Jahr [4]. Zudem wird von den Befürwortern darauf aufmerksam gemacht, dass die Gespräche über die Möglichkeit der Spende mit Angehörigen von Verstorbenen erleichtert würden, wenn die Menschen prinzipiell als willige Spender angesehen würden. Eine Spende wäre unter dieser Voraussetzung der «gewöhnliche» Weg und es könnte in der Tat einfacher sein, zu diesem ja zu sagen als nein, besonders wenn die kurze Entscheidungszeit mit in Betracht gezogen wird.

Tatsächlich haben alle ausser der letzte der genannten Gründe für eine Widerspruchslösung nur Gewicht, wenn die Zahl der Spenderorgane durch dieses Modell tatsächlich erhöht werden könnte. Dies ist auch genau einer der Gründe, warum der Bundesrat das genannte Postulat zurückgewiesen hat. Gemäss seiner Antwort [5] gibt es bis jetzt keine wissenschaftlichen Studien, die hätten zeigen können, ob und wie die verschiedenen Zustimmungsmodelle auf die tatsächliche Anzahl Spenden Einfluss nehmen [3]. 
Die Rechtslage bezüglich der Zustimmung zur Organentnahme variiert von Land zu Land [6]. Deutschland, Dänemark, Grossbritannien, Irland und Holland beispielsweise wenden wie die Schweiz die erweiterte Zustimmungslösung an; Italien, Norwegen, Österreich, Portugal und Spanien benutzen die enge oder erweiterte Widerspruchlösung und Schweden kennt die Informationslösung. In Spanien beispielsweise, wo zwar die Widerspruchslösung gesetzlich verankert ist, faktisch aber die erweiterte Zustimmungslösung angewendet wird, gibt es pro Million Einwohner die weltweit meisten Spenderorgane. Auch in der Schweiz verfügte - vor dem Inkrafttreten des Bundesgesetzes über die Transplantation - ein Kanton (Tessin) mit der erweiterten Zustimmungslösung über die meisten Spenderorgane. Andererseits hat die Schweiz, etwa im Vergleich zu Österreich, eine ziemlich tiefe Spenderate. Obwohl ein Einfluss des Zustimmungsmodells auf die Spendebereitschaft und die tatsächliche Spenderzahl grundsätzlich möglich ist, sind die Unterschiede in den Spenderaten wahrscheinlich in den unterschiedlichen Gesundheitssystemen und den variierenden sozialen, kulturellen und rechtlichen Bedingungen eines Landes oder einer Region zu suchen. In der Schweiz liegt ein Grund für unterschiedliche Spenderaten zwischen Regionen vermutlich in der Art und Weise, wie Spitäler organisiert sind: Die Spitäler, welche die meisten Spender verzeichnen, sind jedes Jahr die gleichen [3].

\section{Eingriff in die persönlichen Rechte}

Es ist sicherlich wünschenswert, rechtliche, institutionelle und soziale Bedingungen zu hinterfragen, um das Ziel einer möglichst hohen Spenderate zu verfolgen. Ist es aber wirklich besser, das Zustimmungsmodell durch das Widerspruchsmodell zu ersetzen, um - vielleicht - mehr Spenderorgane zur Verfügung zu haben? Auch wenn unter der Widerspruchslösung mehr Spenderorgane gewonnen werden könnten, gibt es einen gewichtigen Grund, warum die Zustimmungslösung trotzdem nicht ersetzt werden sollte. Es ist eine prinzipielle Frage, ob die Achtung der persönlichen Rechte der Verfügung über den eigenen (toten) Körper geschützt oder untergraben werden soll. Ein Regimewechsel würde letzterem zu nahe kommen. Es ist paradox, wenn jede Person, die ihre Organe nicht spenden will, sich in ein Register eintragen lassen muss, damit ihr nach dem Tod etwas Ureigenes nicht weggenommen wird. ${ }^{1}$ Wie wäre es, als Beispiel für eine ähnliche Situation, wenn wir ohne informierte Zustimmung einer Person ihren toten Körper für die medizinische Lehre und Forschung benutzen würden? Für die Gesellschaft wäre wahrscheinlich ein zusätzlicher Nutzen da, wenn tote Körper plötzlich in grösserer Zahl verfügbar wären. Wäre es aber angemessen, zu Lebzeiten explizit nein sagen zu müssen, wenn man seinen Körper nach dem Tod nicht zu diesem Zweck verfügbar machen will? Ein toter Körper und ein Organ als dessen Bestandteil sind nichts, worüber eine staatliche oder private Institution ohne aktive Zustimmung verfügen kann. Auch wenn - bei Anwendung der erwei-

1 Eigentum meint hier das persönliche Recht bzw. das Recht der Angehörigen im Rahmen des Persönlichkeitsrechts, über den toten Körper zu verfügen. terten Widerspruchslösung - die Angehörigen oder vertraute Personen über die Organentnahme befragt würden, darf die Entscheidung über die Organspende nicht per Gesetz an Dritte delegiert werden, sondern sie muss kategorisch die persönliche und freiwillige einer jeden einzelnen Person sein. Die Annahme, dass jede Person, die sich zu Lebzeiten nicht zur eigenen Organspende geäussert hat, eine willige Spenderin oder ein williger Spender ist, untergräbt diese persönliche Entscheidung.

Zum Schluss sollen noch zwei mögliche Einwände angesprochen werden, die die Zustimmungslösung zu entkräften versuchen. Erstens wird von den Befürwortern einer Widerspruchslösung immer wieder hervorgebracht, dass die Mehrheit der Bürgerinnen und Bürger in der Schweiz eigentlich bereit wären ihre Organe zu spenden, sich zu Lebzeiten aber nie dazu geäussert haben. Diese sind unter der Zustimmungslösung in der Tat so etwas wie «verlorene» Organe. Es ist aber genauso wahrscheinlich, dass es Menschen gibt, die sich zu einer Organspende nie geäussert haben, dieser aber nicht zustimmen würden, wenn sie gefragt würden. Wären diese aber wiederum, im Falle ihrer Entnahme unter der Widerspruchslösung, nicht unrechtmässig zu Eigen gemachte, um nicht zu sagen, gestohlene Organe? Zudem darf die postulierte Meinung der Mehrheit nicht als Rechtfertigung für die Missachtung der Rechte einer Minderheit benutzt werden. Zweitens gibt es die Auffassung, dass wir sowieso alle moralisch verpflichtet seien, unsere Organe zu spenden. Hätten wir diese Verpflichtung aber nicht nur, wenn es gleichzeitig das Recht auf ein Spenderorgan gäbe? Wir haben aber weder ein gesetzliches, noch ein moralisches Recht auf ein Spenderorgan. Schweigen darf aus den genannten Gründen nicht als Zustimmung gewertet werden. Die Aussicht auf eine höhere Zahl an Spenderorganen vermag den Einsatz für einen Regimewechsel nicht zu rechtfertigen.

\section{Korrespondenz}

Eliane Pfister, lic. phil.

Institut für Biomedizinische Ethik

Universität Zürich

Pestalozzistrasse 24

CH-8032 Zürich

e-mail: pfister@ethik.uzh.ch

\section{Referenzen}

1. http://www.bag.admin.ch/transplantation/06518/06519/index. html?lang=de (aufgerufen am 19.8.2010)

2. http://www.admin.ch/ch/d/sr/810_21/a8.html (aufgerufen am 19.8.2010)

3. Vgl. Neue Zürcher Zeitung. Für Regimewechsel bei der Organspende. 11.09.2009 und Neue Zürcher Zeitung. Widerspruch statt Zustimmung. 23.9.2009.

4. Berner Zeitung, Interview mit Franz Immler, Direktor Swisstransplant: Sparpotenzial von einer Milliarde. 17.08.2009.

5. http://www.parlament.ch/d/suche/seiten/geschaefte.aspx?gesch_ id=20083158 (aufgerufen am 19.8.2010).

6. Die aufgeführten Daten stammen vom Bundesamt für Gesundheit vgl. http://www.bag.admin.ch/transplantation/00694/00727/index. html?lang=de (aufgerufen am 19.8.2010). 